\title{
Manejo Quirúrgico del Queratoquiste Odontogénico con Invasión al Seno Maxilar: Reporte de Serie Casos y Revisión de la Literatura
}

\author{
Surgical Management of Odontogenic Keratocyst with Invasion of \\ the Maxillary Sinus: Cases Report and Review of the Literature
}

Liberto Figueroa C.1,2; Felipe López N.; Melissa Carvajal G..; Sebastián Mordoh C. ${ }^{2}$ \& Francisca Núñez M.1

FIGUEROA, C. L.; LÓPEZ, N. F.; CARVAJAL. G. M.; MORDOH. C. S. \& NÚÑEZ, M. F. Manejo quirúrgico del queratoquiste odontogénico con invasión al seno maxilar: Reporte de serie casos y revisión de la literatura. Int. J. Odontostomat., 15(4):953959, 2021.

RESUMEN: El queratoquiste odontogénico (QQO) es una quiste intraóseo poco frecuente que varía entre un 3 a 11 $\%$ de todos los quistes odontogénicos, su ubicación en el maxilar es rara y la invasión al seno maxilar lo es aún más. El QQO es una patología benigna, localmente agresiva que tiene una alta tasa de recidiva. Se han descrito diversas técnicas quirúrgicas para su tratamiento, las cuales van desde lo más conservador como la enucleación a lo más radical como una resección. El uso de agentes coadyuvantes químicos o cauterizantes han logrado disminuir la tasa de recidiva en conjunto con tratamientos más conservadores, disminuyendo la morbilidad y secuelas asociada a una resección. El objetivo de este trabajo es presentar una serie de casos clínicos de QQO que invaden el seno maxilar, su tratamiento de manera conservadora y una revisión de la literatura comparando los diversos tratamientos y su tasa de recidiva.

PALABRAS CLAVE: queratoquiste, seno maxilar, solución de carnoy, nitrógeno líquido, descompresión.

\section{INTRODUCCIÓN}

El Queratoquiste Odontogénico (QQO), Ex tumor Odontogénico Queratinizante Quístico, vuelve a ser considerada una lesión quística según la OMS el 2017 (Westra \& Lewis, 2017). Uno de los rasgos característicos de esta patología es la tendencia a crecer a lo largo de los canales esponjosos con muy poca expansión cortical (Menon, 2015). Se han propuesto varias teorías de expansión, estas incluyen hiperosmolaridad intraluminal, proliferación epitelial activa (Toller, 1967), actividad colagenolítica de la pared del quiste (Ahlfors et al., 1984) y síntesis de interleucina 1 y 6 por los queratinocitos. Esto inducirá la secreción del factor de crecimiento de queratinocitos de los fibroblastos interactivos junto con el factor de necrosis tumoral, lo que provocará un aumento de los niveles de prostaglandinas y una mayor expresión de la proteína relacionada con las paratiroides. También se ha visto que la liberación de citocinas inflamatorias como IL-1 de las células epiteliales tienden a activar la reabsorción del hueso alrededor de las lesiones estimulando la activación de la osteoclastogénesis. (Barnes et al., 2005).

Tiene un carácter localmente agresivo y presenta una tasa de recidiva la cual varía ampliamente entre un $5 \%$ al $70 \%$ (Hyun et al., 2009). En un estudio retroespectivo realizado por Sánchez-Burgos et al. (2014) concluyeron que la tasa de recidiva fue del 25 $\%$ tras un seguimiento medio de 5 años. No se encontró asociación significativa con recidiva respecto al sexo o edad. Los QQO suelen ser lesiones mandibulares uniquísticas de origen odontógeno. La mayoría de ellos

\footnotetext{
${ }^{1}$ Asignatura de Cirugía bucal y maxilofacial, Facultad de Salud y Odontología, Universidad Diego Portales, Santiago, Chile.

${ }^{2}$ Asignatura de Cirugía y traumatología bucomaxilofacial, Facultad de Medicina Clinica Alemana- Universidad del Desarrollo, Santiago, Chile.
} 
FIGUEROA, C. L.; LÓPEZ, N. F.; CARVAJAL. G. M.; MORDOH. C. S. \& NÚÑEZ, M. F. Manejo quirúrgico del queratoquiste odontogénico con invasión al seno maxilar: Reporte de serie casos y revisión de la literatura. Int. J. Odontostomat., 15(4):953-959, 2021.

están revestidos con epitelio escamoso paraqueratinizado y algunos están ortoqueratinizados. El tipo paraqueratinizado tiene una alta tasa de recurrencia, un carácter localmente destructivo y una tendencia a ser múltiple (Dereci et al., 2011).

El tratamiento ha sido controversial debido al potencial de recurrencia del quiste. El objetivo del tratamiento del QQO debe ser eliminar la lesión con menor riesgo posible de recidiva y mínima morbilidad. Las opciones de manejo en QQO se pueden dividir ampliamente en tratamiento conservador y manejo radical. El tratamiento conservador tiene como objetivo preservar la arquitectura ósea tanto como sea posible mientras se elimina la patología, como alternativa conservadora se describe la descompresión sin embargo, presenta cifras de recidivas que pueden llegar a un $56 \%$. Dentro de las alternativas quirúrgicas para el tratamiento se describen técnicas quirùrgicas-conservadoras tales como la enucleación con/sin legrado óseo junto con usos complementarios de cauterización química/criogénica. (Meno, 2015).

Se han propuesto y utilizado ampliamente algunos tratamientos coadyuvantes a la enucleación como la solución de Carnoy y nitrógeno líquido para así realizar cirugías menos invasivas. En una revisión de la literatura, evidenció que la enucleación más solución de Carnoy, con o sin ostectomía periférica, da como resultado una tasa de recidiva de un $8 \%$. El uso de crioterapia con enucleación resulta en una tasa de recurrencia de un 38 \%. La resección marginal, a pesar de una tasa de recurrencia de $0 \%$, no es estadísticamente significativa mejor para eliminar las recidivas que la enucleación más la solución de Carnoy. Por lo tanto, para minimizar la invasividad y la recidiva, la opción de tratamiento más eficaz parece ser la enucleación del QQO y la aplicación posterior de la solución de Carnoy. (Madras \& Lapointe, 2008)

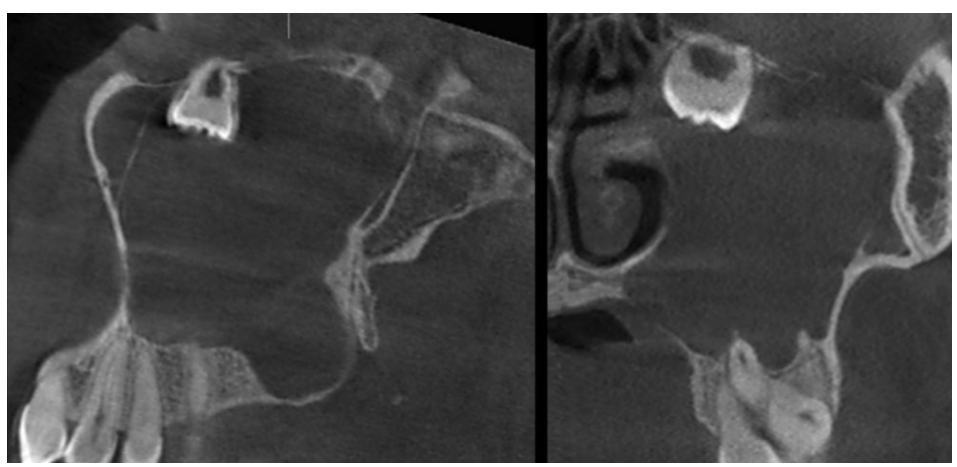

Fig. 1. En TAC sagital y frontal se observa $Q Q O$ ocupando todo el seno maxilar y diente 2.8 ectópico bajo el piso de la órbita.

\section{CASOS CLÍNICOS}

Caso 1. Paciente de 19 años, sin antecedentes mórbidos, consultó en marzo del año 2013 con un cirujano maxilofacial, por sensación de plenitud en el maxilar izquierdo, dolor a la palpación en relación al seno maxilar izquierdo. Se realizó una tomografía axial computarizada (TAC) de cara y se observó una gran lesión osteolítica que ocupaba parcialmente el seno maxilar izquierdo, pérdida de tabla ósea lateral y desplazamiento diente 2.8 hacia el piso orbitario (Fig. 1).

Se realizó una biopsia mediante punción aspirativa, resultando ser un queratoquiste odontogénico paraqueratinizado. Se colocó una cánula para descomprimir la lesión antes de realizar cirugía resectiva.

Tras un año postoperatorio, al examen imagenológico se observó una extensa lesión osteolítica en seno maxilar izquierdo, corticalizada que se extendía próximo a piso de órbita hasta ápices dentarios, entre dientes 2.6 a zona de la tuberosidad. Se observó perforación cortical entre dientes 2.6 - 2.7 a nivel apical y sobre apical por delante del proceso cigomático alveolar y expansión con adelgazamiento de tabla vestibular con leves perforaciones en zona de la tuberosidad. Se realizó exéresis del QQO, desinclusión de tercer molar y aplicación de nitrógeno líquido en las paredes óseas de la lesión, bajo anestesia general.

La paciente retomó controles 4 años postoperatorio, se tomó TAC de control y se verificó permanencia del volumen del quiste, nuevamente se opta por descompresión del quiste.

En control Marzo del 2020, se observó en TAC, disminución del tamaño de la lesión y neoformación de tejido óseo en pared lateral y posterior de aspecto grueso de seno maxilar izquierdo (Fig. 2).

En diciembre del 2020, bajo anestesia general, se realizó la quistectomía más aplicación de solución de Carnoy en gasa durante 90 segundos, se lavó profusamente la cavidad con suero fisiológico. Se envió muestra a estudio histopatológico que confirmó el diagnóstico de queratoquiste odontogénico paraqueratinizado. La paciente evolucionó de forma favorable. Se realizó estudio al mes postoperatorio sin hallazgos. 


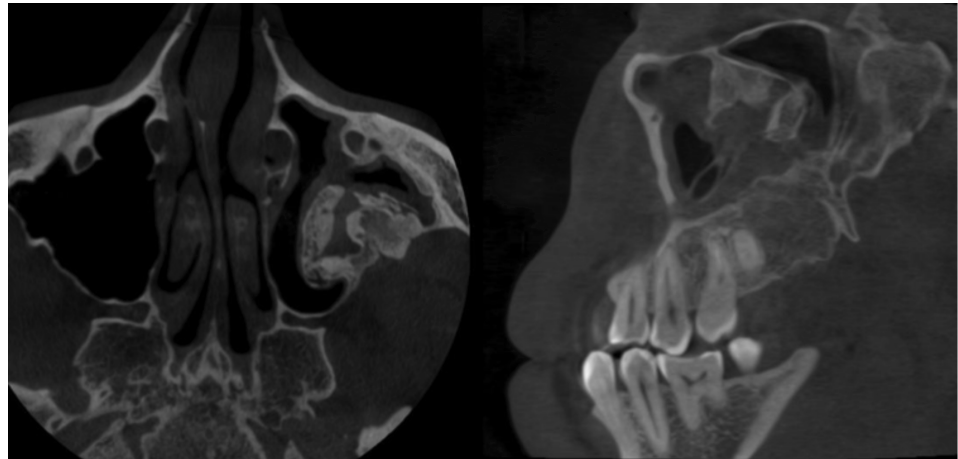

Fig. 2. En TAC sagital y axial se puede observar gran ocupación y calcificación del seno maxilar izquierdo por el QQO.

Caso 2. Paciente de 12 años sexo masculino, sin antecedentes mórbidos, consultó por hallazgo radiográfico el cual presentaba una lesión destructiva del maxilar, reabsorción corticales en relación a dientes 1.1 a 1.4 , se exteriorizaba en vestíbulo e invadía seno maxilar y fosa nasal derecha, relacionado a diente 1.3 incluido. Se realizó biopsia incisional dando como resultado QQO ortoqueratinizado. Se realizó enucleación, legrado, aplicación de solución de Carnoy y desinclusión de diente 1.3. Se controló durante 12 años sin presencia de recidiva (Fig. 3 ).

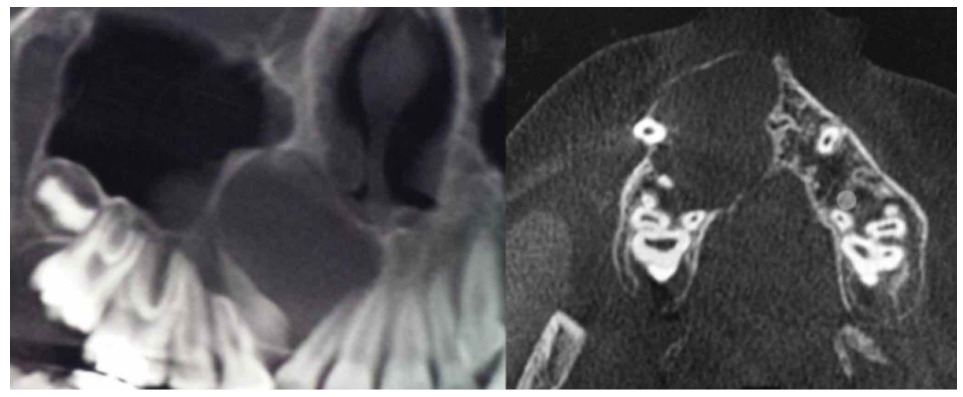

Fig. 3. Se observa $Q Q O$ en seno maxilar derecho relacionado a diente 1.3 incluido.

Caso 3. Paciente de 64 años de sexo masculino, consultó por hallazgo radiográfico de lesión radiolúcida en relación a diente 1.8 incluido. Se definió realizar biopsia incisional dando como resultado QQO paraqueratinizado. Se determinó realizar
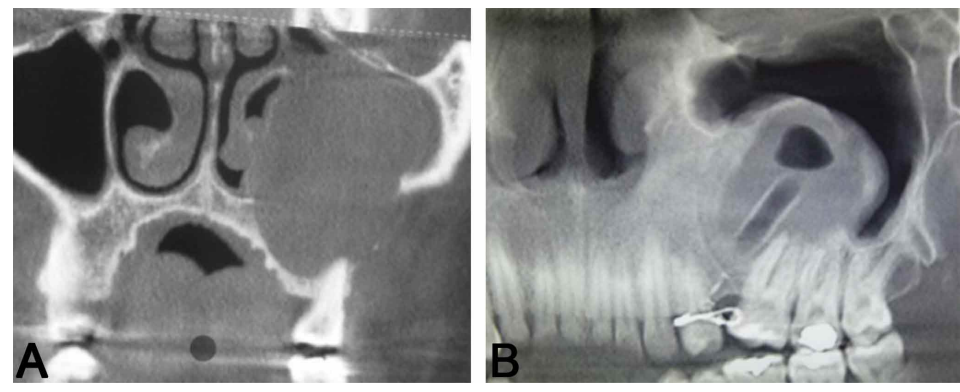

desinclusión de diente 1.8, enucleación de la lesión, legrado óseo y aplicación de crioterapia (nitrógeno líquido). Se ha controlado por 2 años, sin indicios de recidiva (Fig. 4).

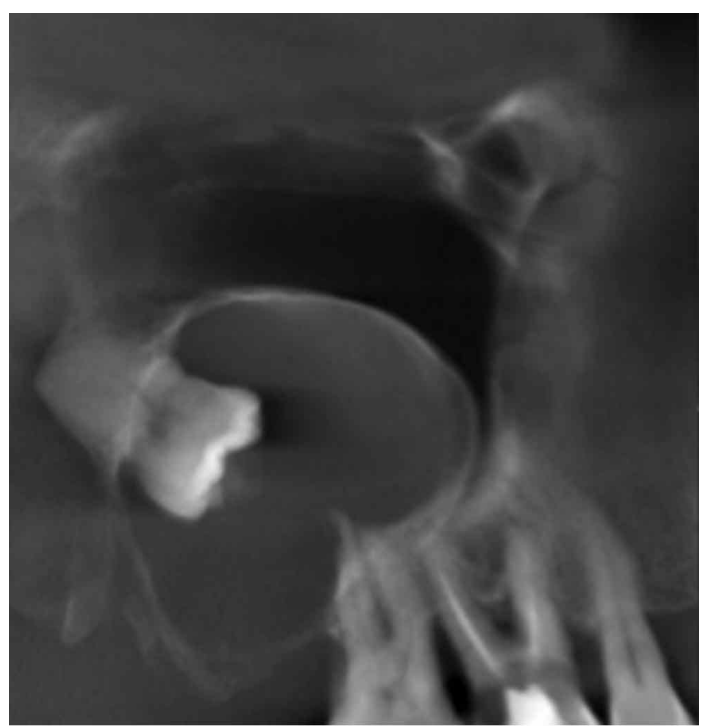

Fig. 4. Se observa $Q Q O$ que invade el seno maxilar derecho en relación a diente 1.8 incluido en posición ectópica.

Caso 4. Paciente de 26 años de sexo masculino, consultó por hallazgo en radiografía retroalveolar de lesión radiolúcida en relación a diente 2.5 incluido, se realizó biopsia incisional y descompresión con un dren por 6 meses (Fig. 5).

El estudio histopatológico determinó QQO paraqueratinizado. Se realizó enucleación, legrado óseo y aplicación de solución de Carnoy. Sin seguimiento.

Los casos se encuentran resumidos en la Tabla I.

\section{DISCUSIÓN}

Fig. 5. A. Se observa seno maxilar izquierdo completamente ocupado por QQO. B Presencia de cánula de descompresión y seno maxilar con lesión en franca disminución. 
FIGUEROA, C. L.; LÓPEZ, N. F.; CARVAJAL. G. M.; MORDOH. C. S. \& NÚÑEZ, M. F. Manejo quirúrgico del queratoquiste odontogénico con invasión al seno maxilar: Reporte de serie casos y revisión de la literatura. Int. J. Odontostomat., 15(4):953-959, 2021.

Tabla I. Resumen de los casos expuestos en cuanto a sexo, edad, dientes incluidos, tratamiento, recidiva y seguimiento.

\begin{tabular}{cccccccc}
\hline aso & Sexo & Edad (años) & $\begin{array}{c}\text { Dientes } \\
\text { incluidos }\end{array}$ & Descompresión & Tratamiento & Recidiva & Seguimiento \\
\hline 1 & $\mathrm{M}$ & 19 & 2.8 & $\mathrm{Si}$ & $\mathrm{E}+\mathrm{L}+\mathrm{C}$ y SC & Si & 5 meses \\
2 & $\mathrm{M}$ & 12 & 1.3 & No & E+L+SC & No & 12 años \\
3 & $\mathrm{M}$ & 64 & 1.8 & $\mathrm{E}+\mathrm{L}+\mathrm{C}$ & No & 2 años \\
4 & $\mathrm{M}$ & 26 & 2.5 & $\mathrm{Si}$ & $\mathrm{E}+\mathrm{L}+\mathrm{SC}$ & NA & NA \\
\hline
\end{tabular}

$\mathrm{M}=$ masculino, $\mathrm{E}=$ enucleación, $\mathrm{C}=$ crioterapia, $\mathrm{SC}=$ solución de Carnoy, $\mathrm{NA}=$ no informado.

Los QQO con invasión del seno maxilar es poco frecuente, con menos del $1 \%$ de casos reportados en la literatura (Silva et al., 2006). El seno maxilar forma parte de los senos paranasales, en proximidad con los ápices dentarios. Propenso a infecciones, quistes y tumores de origen odontogénico. El diagnóstico de dicha lesión es un desafío ya que las radiografías maxilares no ofrecen signos característicos debido a la superposición de varias estructuras (Cioffi et al., 1987). Puede ocurrir una patología del seno maxilar cuando la membrana de Schneider se rompe por condiciones como la patología odontogénica del hueso maxilar. Las infecciones y patologías odontogénicas representan entre el 11 $\%$ y el $12 \%$ de los casos de sinusitis maxilar (Cakur et al., 2008).

Aproximadamente el $30 \%$ de los QQO están asociados con al menos un diente no erupcionado, más comúnmente los terceros molares (Chirapathomsakul et al., 2006). En nuestra serie de casos se presentan dos pacientes con terceros molares incluidos involucrados.

Histológicamente se pueden diferenciar según su queratinización la cual es predominantemente paraqueratósica, pero puede ser ortoqueratótica. La variante ortoqueratinizada tiene un potencial de crecimiento limitado, baja tasa de recidiva y no se asocia con el síndrome de Gorlin, por lo tanto, debe distinguirse de la variante paraqueratinizada (Chi et al., 2005).

Se ha informado sobre el comportamiento agresivo de algunos $Q Q O$ en la medida en que han penetrado el hueso cortical y han afectado los tejidos blandos circundantes (Emerson et al., 1972).

Los QQO de gran tamaño en el maxilar pueden afectar el seno maxilar, causar la destrucción del piso de la órbita y proptosis de los globos oculares (Kshirsagar et al., 2019).

Se ha propuesto que las características invasivas del QQO se deben a la presencia de receptor kappa B nuclear activador (RANK) en el epitelio del quiste. RANK es un activador central del factor de necrosis kappa B, que controla la transcripción del ADN y es el receptor de señalización del ligando RANK (RANKL). RANKL se une a RANK en la superficie de los preosteoclastos y estimula el desarrollo y la activación de los osteoclastos (Lacy et al., 1998). Se sugiere que RANK se origina en el epitelio odontogénico, aumentando la reabsorción ósea debido a su efecto sobre el crecimiento del quiste, la proliferación celular o el retraso de la apoptosis (Da Silva et al., 2008).

En el estroma de los QQO se ha identificado la presencia de MMP2. Los QQO tienden a formar quistes satélites a través del desprendimiento del revestimiento de células epiteliales de la pared del tejido conectivo. Se ha pensado que este comportamiento molecular es responsable de las recidivas frecuentes luego de la enucleación quirúrgica (Sternlicht \& Werb, 2001). La reabsorción ósea mediada por la activación de la vía de señalización RANK/RANKL y la degradación de la matriz por activación de la vía MMP2 en el QQO identifica su comportamiento biológico agresivo. Por lo tanto la alta expresión de MMP2, RANK y RANKL está relacionada con el comportamiento biológico de esta entidad (Al-Rawi et al., 2018).

Se desconoce el mecanismo patológico de formación de hueso tan cerca del epitelio a pesar de la presencia de inflamación. Los elementos epiteliales de los tumores odontogénicos expresan activamente la sialoproteína ósea (BSP) que puede desempeñar un papel importante en la formación y diferenciación tumoral con respecto a la calcificación patológica (Chen et al., 1998). El componente epitelial odontogénico puede expresar BSP y esto podría explicar la presencia de tejido óseo en la pared de tejido fibroso de los QQO, que es una ocurrencia extremadamente rara (Gotmare et al., 2016).

En el presente caso clínico, como secuencia de tratamiento, se realizó una descompresión la cual tiene como objetivo realizar un tratamiento conservador, en donde se posiciona un dispositivo (dren) el cual es utili- 
FIGUEROA, C. L.; LÓPEZ, N. F.; CARVAJAL. G. M.; MORDOH. C. S. \& NÚÑEZ, M. F. Manejo quirúrgico del queratoquiste odontogénico con invasión al seno maxilar: Reporte de serie casos y revisión de la literatura. Int. J. Odontostomat., 15(4):953-959, 2021.

zado para reducir el tamaño del quiste y reforzar la cortical fina, realizándose posteriormente un legrado para verificar la regeneración ósea y la no recidiva de la lesión (Pogrel, 2005). Se ha demostrado una resolución completa tanto clínica como radiográfica después de la descompresión con un seguimiento medio de 2,8 años. Aunque se puede lograr la resolución completa de la lesión mientras se preserva la anatomía y la función, es mandatorio realizar un seguimiento a largo plazo de estos pacientes, porque se ha informado que la recidiva ocurre hasta 10 años después del tratamiento (Kolokythas et al., 2007).

La crioterapia con nitrógeno líquido consiste en eliminar el tejido patológico visible y necrosar, mediante la congelación, los posibles restos celulares que pueden conducir a la recidiva. El nitrógeno líquido tiene propiedades necrotizantes celulares y preserva las estructuras óseas inorgánicas, a diferencia de la solución de Carnoy, que destruye las propiedades osteogénicas y osteoconductivas. Esta técnica de crioterapia preserva la estructura ósea y da como resultado una mejor reparación (Tonietto et al., 2011). En una revisiónsistemática en donde se analizaron 35 estudios donde se trataron 2287 QQO reportaron una tasa de recidiva al realizar enucleación junto a crioterapia del 14,5 \% (Al-Moraissi et al., 2017), aunque otra revisión sistemática ha reportado un $38 \%$ de recidiva (Madras \& Lapointe).

En los $Q Q$ que presentan recidiva, se deben buscar alternativas de coadyuvantes que presentan menor tasa de recidiva como la solución de Carnoy, la cual penetra en los espacios esponjosos del hueso y, por lo tanto, desvitaliza y fija las células tumorales restantes. Se cree que el éxito de la aplicación de este agente después de la enucleación se debe tanto a la acción de penetración como a la de fijación (Lau \& Samman, 2006). Se puede aplicar dentro de la lesión quística o, más comúnmente, directamente sobre el lecho óseo restante después de que se haya eliminado la lesión. Se pensó que el éxito de la aplicación de este agente después de la enucleación se debía tanto a su acción de penetración como a su acción de fijación. La práctica habitual es aplicar la solución de Carnoy con aplicadores de algodón o gasa durante 3-5 minutos, y lavar el defecto óseo. Dentro de las complicaciones descritas por el uso de la solución de Carnoy se describen infección, dehiscencias, formación de secuestro óseo y neuropatía (Ribeiro-Júnior et al., 2007). Frerich et al. (1994) sugirieron que la aplicación de la solución de Carnoy no debe exceder de 3-5 min. Demostraron que el tiempo crítico hasta la afectación nerviosa del nervio alveolar inferior del conejo era de 3-5 min, y que la solución de Carnoy no debería aplicarse directamente sobre el nervio debido a su composición neurotóxica. Se concluye que cualquier daño ocurrido en los vasos sanguíneos es reversible cuando los tiempos de exposición son $<5 \mathrm{~min}$ (Frerich et al., 1994).

Se decidió utilizar este coadyuvante ya que se quería seguir manteniendo el tratamiento conservador pero con una solución que reportará menor tasa de recidiva que la crioterapia. Se ha reportado una tasa de recidiva que según revisiones sistemáticas van desde el 1,6 \% al 11,5\% (Blanas et al., 2000; Al-Moraissi et al.), la cual es considerablemente menor a lo reportado con crioterapia, pero como se comentó anteriormente con mayores complicaciones que la crioterapia.

En la revisión de literatura (Tabla II), se resume el porcentaje de recurrencia reportado, comparando el uso del nitrógeno líquido y la solución de Carnoy.

Tabla II. Resumen de artículos revisados en cuanto a porcentaje de recidiva comparando el uso de nitrógeno líquido y solución de Carnoy.

\begin{tabular}{lccc}
\hline Autores & Año & $\begin{array}{c}\text { Recidiva } \\
\text { E+C }\end{array}$ & $\begin{array}{c}\text { Recidiva } \\
\text { E+SC }\end{array}$ \\
\hline Madras \& Lapointe & 2008 & $38 \%$ & $8 \%$ \\
Al-Moraisi et al. & 2017 & $14.4 \%$ & $11,5 \%$ \\
Santos De Castro et al. & 2018 & $11,5 \%$ & $7,8 \%$ \\
Chrcanovic et al. & 2017 & $20,9 \%$ & $5,3 \%$ \\
Johnson et al. & 2013 & $30,8 \%$ & $4,8 \%$ \\
Blanas et al. & 2000 & $31,3 \%$ & $1,6 \%$
\end{tabular}

$\mathrm{E}=$ enucleación, $\mathrm{C}=$ crioterapia, $\mathrm{SC}=$ solución de Carnoy.

Como lo han demostrado las diferentes revisiones sistemáticas mostradas en la Tabla II, la tasa recidiva en todas las revisiones es menor al aplicar la solución de Carnoy que utilizando la crioterapia.

Creemos que es importante realizar una descompresión previa a QQO de gran tamaño, para disminuir la morbilidad y tener una cirugía con menor dificultad, ya que cuando el QQO invade el seno maxilar, aumenta la complejidad quirúrgica debido a la dificultad del acceso para lograr enuclear completamente el quiste sin dañar estructuras vecinas importantes, como en el caso 1 que el QQO estaba próximo al piso de la órbita.

En los casos presentados, las raíces de los dientes vecinos se encontraban indemnes y presentaban un diente incluido ectópico, por lo que es importante desincluirlos junto con la enucleación del QQO. 
FIGUEROA, C. L.; LÓPEZ, N. F.; CARVAJAL. G. M.; MORDOH. C. S. \& NÚÑEZ, M. F. Manejo quirúrgico del queratoquiste odontogénico con invasión al seno maxilar: Reporte de serie casos y revisión de la literatura. Int. J. Odontostomat., 15(4):953-959, 2021.

\section{CONCLUSIÓN}

Debido al diferente comportamiento agresivo entre el QQO paraqueratinizado y ortoqueratinizado, se debe conocer con certeza su histopatología antes de realizar el tratamiento quirúrgico.

Cuando nos encontramos con un QQO de gran tamaño recomendamos realizar descompresión, enucleación y aplicación de solución de Carnoy, en especial en el tipo histológico paraqueratinizado, debido a su comportamiento más agresivo y con mayor tasa de recidiva.

Es mandatorio realizar un seguimiento del paciente de al menos 10 años, debido a la alta recidiva que se ha reportado en los QQO.

FIGUEROA, C. L.; LÓPEZ, N. F.; CARVAJAL. G. M.; MORDOH. C. S. \& NÚÑEZ, M. F. Surgical management of odontogenic keratocyst with invasion of the maxillary sinus: Cases report and review of the literature. Int. J. Odontostomat., 15(4):953-959, 2021.

ABSTRACT: Odontogenic keratocyst $(\mathrm{OC})$ is a rare intraosseous pathology that varies between $3 \%$ and $11 \%$ of all odontogenic cysts, its location in the maxilla is rare, and invasion of the maxillary sinus is even more so. OC is a benign, locally aggressive pathology that has a high recurrence rate. Various surgical techniques have been described for its treatment, ranging from the most conservative, such as enucleation, to the most radical, such as resection. The use of chemical or cauterizing adjuvant agents has managed to reduce the recurrence rate in conjunction with more conservative treatments, reducing the morbidity and sequelae associated with a resection. The objective of this work is to present a series of clinical cases of $\mathrm{OC}$ that invade the maxillary sinus, their treatment being carried out in a conservatively manner, and a review of the literature comparing the various treatments and their recurrence rate.

KEY WORDS: keratocyst, maxillary sinus, carnoy's solution, liquid nitrogen, decompression.

\section{REFERENCIAS BIBLIOGRÁFICAS}

Ahlfors, E.; Larsson, A. \& Sjogren, S. The odontogenic keratocyst: a benign cystic tumour? J. Oral Maxillofac. Surg., 42(1):10, 1984.

Al-Moraissi, E. A.; Dahan, A. A.; Alwadeai, M. S.; Oginni, F. O.; AlJamali, J. M.; Alkhutari, A. S.; Al-Tairi, N. H.; Almaweri, A. A. \& AlSanabani, J. S. What surgical treatment has the lowest recurrence rate following the management of keratocystic odontogenic tumor?: A large systematic review and metaanalysis. J. Craniofac. Surg., 45(1):131-44, 2017.

Al-Rawi, N. H.; Al-Siraj, A. K. \& Majeed, A. H. Comparison of osteoclastogenesis and local invasiveness of ameloblastoma and keratocystic odontogenic tumor. Eur. J. Dent., 12(1):36-42, 2018.

Barnes, L.; Eveson, J. W.; Reichart, P. \& Sidransky, D. Pathology and Genetics of Head and Neck Tumours. WHO Classification of Tumour Series. Lyon, IARC Press, 2005.

Blanas, N.; Freund, B.; Schwartz, M. \& Furst, I. M. Systematic review of the treatment and prognosis of the odontogenic keratocyst. Oral Surg. Oral Med. Oral Pathol. Oral. Radiol. Endod., 90(5):5538, 2000.

Cakur, B.; Miloglu, O.; Yolcu, U.; Göregen, M. \& Gürsan, N. Keratocystic odontogenic tumor invading the right maxillary sinus: A case report. J. Oral Sci., 50:345-9, 2008.

Chen, J.; Aufdemorte, T. B.; Jiang, H; Liu, A. R.; Zhang, W. \& Thomas, H. F. Neoplastic odontogenic epithelial cells express bone sialoprotein. Histochem. J., 30:1-6, 1998.

Chi, A. C.; Owings, J. R. \& Muller, S. Peripheral odontogenic keratocyst: report of two cases and review of the literature. Oral Surg. Oral Med. Oral Pathol. Oral Radiol. Endod., 99:71-8, 2005.

Chirapathomsakul, D.; Sastravaha, P. \& Jansisyanont, P. A review of odontogenic keratocysts and the behavior of recurrences. Oral Surg. Oral Med. Oral Pathol. Oral Radiol. Endod., 101:5-9, 2006.

Chrcanovic, B. R. \& Gomez, R. S. Recurrence probability for keratocystic odontogenic tumors: An analysis of 6427 cases. J. Craniofac. Surg., 45(2):244-51, 2017.

Cioffi, G. A.; Terezhalmy, G. T. \& Del Balso, A. M. Odontogenic keratocyst of the maxillary sinus. Oral Surg. Oral Med. Oral Pathol., 64:648-51, 1987.

Da Silva, T. A.; Batista, A. C.; Mendonça, E. F.; Leles, C. R.; Fukada, S. \& Cunha, F. Q. Comparative expression of RANK, RANKL, and $O P G$ in keratocystic odontogenic tumors, ameloblastomas, and dentigerous cysts. Oral Surg. Oral Med. Oral Pathol. Oral Radiol. Endod., 105:333-41, 2008.

Dereci, O.; Oztürk, A. \& Günhan, O. The efficacy of fine needle aspiration cytology in the preoperative evaluation of parakeratotic odontogenic keratocysts. Acta Cytol., 55(2):131-4, 2011.

Emerson, T. G.; Whitlock, R. I. H. \& Jones, J. M. Involvement of soft tissues by odontogenic keratocyst (primordial cyst). Br. J. Oral Surg., 9:181-5, 1972.

Frerich, B.; Cornelius, C. P. \& Wiethölter, H. Critical time of exposure of the rabbit inferior alveolar nerve to Carnoy's solution. J. Oral Maxillofac. Surg., 52:599-606, 1994.

Gotmare, S. S.; Tamgadge, A.; Pereira, T. \& Shetty, A. Keratocystic odontogenic tumor with ossification and calcification: A case report with unusual histological findings. Indian J. Dent. Res., $27: 441-4,2016$

Hyun, H. K.; Hong, S. D. \& Kim, J. W. Recurrent keratocystic odontogenic tumor in the mandible: a case report and literature review. Oral Surg. Oral Med. Oral Pathol. Oral Radiol. Endod., 108:e7-10, 2009.

Johnson, N. R.; Batstone, M. D. \& Savage, N. W. Management and recurrence of keratocystic odontogenic tumor: a systematic review. Oral Surg. Oral Med. Oral Pathol. Oral Radiol., 116(4):e271-6, 2013.

Kolokythas, A.; Fernandes, R. P.; Pazoki, A. \& Ord, R. A. Odontogenic keratocyst: to decompress or not to decompress? A comparative study of decompression and enucleation versus resection/ peripheral ostectomy. J. Oral Maxillofac. Surg. 65(4):640-4, 2007.

Kshirsagar, R. A.; Bhende, R. C.; Raut, P. H.; Mahajan, V.; Tapadiya, V. J. \& Singh, V. Odontogenic keratocyst: developing a protocol for surgical intervention. Ann. Maxillofac. Surg. 9(1):152-7, 2019.

Lacy, D. L.; Timms, E.; Tan, H. L.; Kelley, M. J.; Dunstan, C. R. \& Burgess, T. Osteoprotegerin ligand is a cytokine that regulates osteoclasts differentiation and activation. Cell, 93:165-76, 1998. 
FIGUEROA, C. L.; LÓPEZ, N. F.; CARVAJAL. G. M.; MORDOH. C. S. \& NÚÑEZ, M. F. Manejo quirúrgico del queratoquiste odontogénico con invasión al seno maxilar: Reporte de serie casos y revisión de la literatura. Int. J. Odontostomat., 15(4):953-959, 2021.

Lau, S. L. \& Samman, N. Recurrence related to treatment modalities of unicystic ameloblastoma: a systematic review. J. Oral Maxillofac. Surg., 35:681-90, 2006.

Madras, J. \& Lapointe, H. Keratocystic odontogenic tumour: reclassification of the odontogenic keratocyst from cyst to tumour. J. Can. Dent. Assoc., 74(2):165-165h, 2008.

Menon, S. Keratocystic odontogenic tumours: etiology, pathogenesis and treatment revisited. J. Maxillofac. Oral Surg., 14(3):541-7, 2015

Pogrel, M. A. Treatment of keratocysts: the case for decompression and marsupialization. J. Oral Maxillofac Surg., 63:1667-73, 2005.

Ribeiro-Júnior, O.; Borba, A. M.; Alves, C. A. \& Guimãraes-Júnior, J. Complications of Carnoy solution in the treatment of odontogenic tumors. RGO, 55:263-6, 2007.

Sánchez-Burgos, R.; González-Martín-Moro, J.; Pérez-Fernández, E. \& Burgueño-García, M. Clinical, radiological and therapeutic features of keratocystic odontogenic tumours: a study over a decade. J. Clin. Exp. Dent., 6(3):e259-e264, 2014.

Santos De Castro, M.; Caixeta, C. A.; De Carli, M. L.; Ribeiro-Júnior, N. V.; Miyazawa, M.; Pereira, A. C.; Sperandio, F. F. \& Hanemann, J. A. Conservative surgical treatments for nonsyndromic odontogenic keratocysts: a systematic review and meta-analysis. Clin Oral Investig., 22(5):2089-101, 2018.

Silva, G. C.; Silva, E. C.; Santiago-Gomez, R. \& Vieira, T. C. Odontogenic keratocyst in the maxillary sinus: Report of two cases. Oral Oncol., 42:231-4, 2006.

Sternlicht, M. D. \& Werb, Z. How matrix metalloproteinases regulate cell behavior. Rev. Cell. Dev. Biol., 17:463-516, 2001.

Toller, P. Origin and growth of cysts of the jaws. Ann. R. Coll. Surg. Engl., 40(5):306-36, 1967

Tonietto, L.; Borges, H. O.; Martins, C. A.; Silva, D. N. \& Sant'AnaFilho, M. Enucleation and liquid nitrogen cryotherapy in the treatment of keratocystic odontogenic tumors: a case series. J. Oral Maxillofac Surg., 69(6):e112-7, 2011.

Westra, W. H. \& Lewis, J. S. Update from the 4th Edition of the World Health Organization Classification of Head and Neck Tumours: Oropharynx. Head Neck Pathol., 11(1):41-7, 2017.
Corresponding author:

Felipe López

Facultad de Salud y Odontología

Universidad Diego Portales

Santiago

CHILE

E-mail: felipe.lopezn@mail.udp.cl 